\title{
Science of Mini-EUSO detector on board the International Space Station
}

\author{
M. Casolino*1, A. Belov ${ }^{2}$, M. Bertaina $^{5}$, G. Cambie' ${ }^{3}$, F. Capel ${ }^{4}$, T. Ebisuzaki ${ }^{6}$, P. \\ Klimov $^{2}$, M. Panasyuk ${ }^{2}$, P. Picozza ${ }^{3}$, M. Ricci ${ }^{7}$, \\ for the JEM-EUSO collaboration \\ ${ }^{1}$ INFN and RIKEN, ${ }^{2}$ Lomonosov Moscow State University, Skobeltsyn Institute of Nuclear \\ Physics (SINP MSU), Moscow, Russia, ${ }^{3}$ University of Rome Tor Vergata, Rome, ${ }^{4} \mathrm{KTH}$, Royal \\ Institute of Technology, Stockholm - Sweden, ${ }^{5}$ INFN and University of Torino, Italy, ${ }^{6}$ Riken, \\ Japan, ${ }^{7}$ INFN LNF, Italy \\ E-mail: casolino@roma2.infn.it
}

\begin{abstract}
The Mini-EUSO space experiment ("UV atmosphere" in Russian Space Program) is a telescope designed to perform observations of night-time Earth in the UV spectrum. The instrument comprises a compact telescope with a large field of view $\left(44^{\circ} \times 44^{\circ}\right)$, based on an optical system employing two $25 \mathrm{~cm}$ diameter Fresnel lenses (focal length $\simeq 30 \mathrm{~cm}$ ) for increased light collection. Mini-EUSO will study different scientific phenomena ranging from strange quark matter and Ultra High Energy Cosmic Rays (UHECRs) to bioluminescence and atmospheric physics. It will also create the first night-time map of the Earth in UV light. The mission will raise the technology readiness level (TRL) of the future JEM-EUSO missions to observe UHECRs from space. The Mini-EUSO measurements will be performed from the ISS through a UV transparent window in the Russian Zvezda Service Module.

Launch is foreseen between Autumn 2017 and beginning 2018 in the framework of the next manned ASI (Italian Space Agency) flight and observations are supposed to continue with Russian cosmonauts for several years.
\end{abstract}

35th International Cosmic Ray Conference - ICRC2017

10-20 July, 2017

Bexco, Busan, Korea

${ }^{*}$ Speaker. 


\section{Introduction}

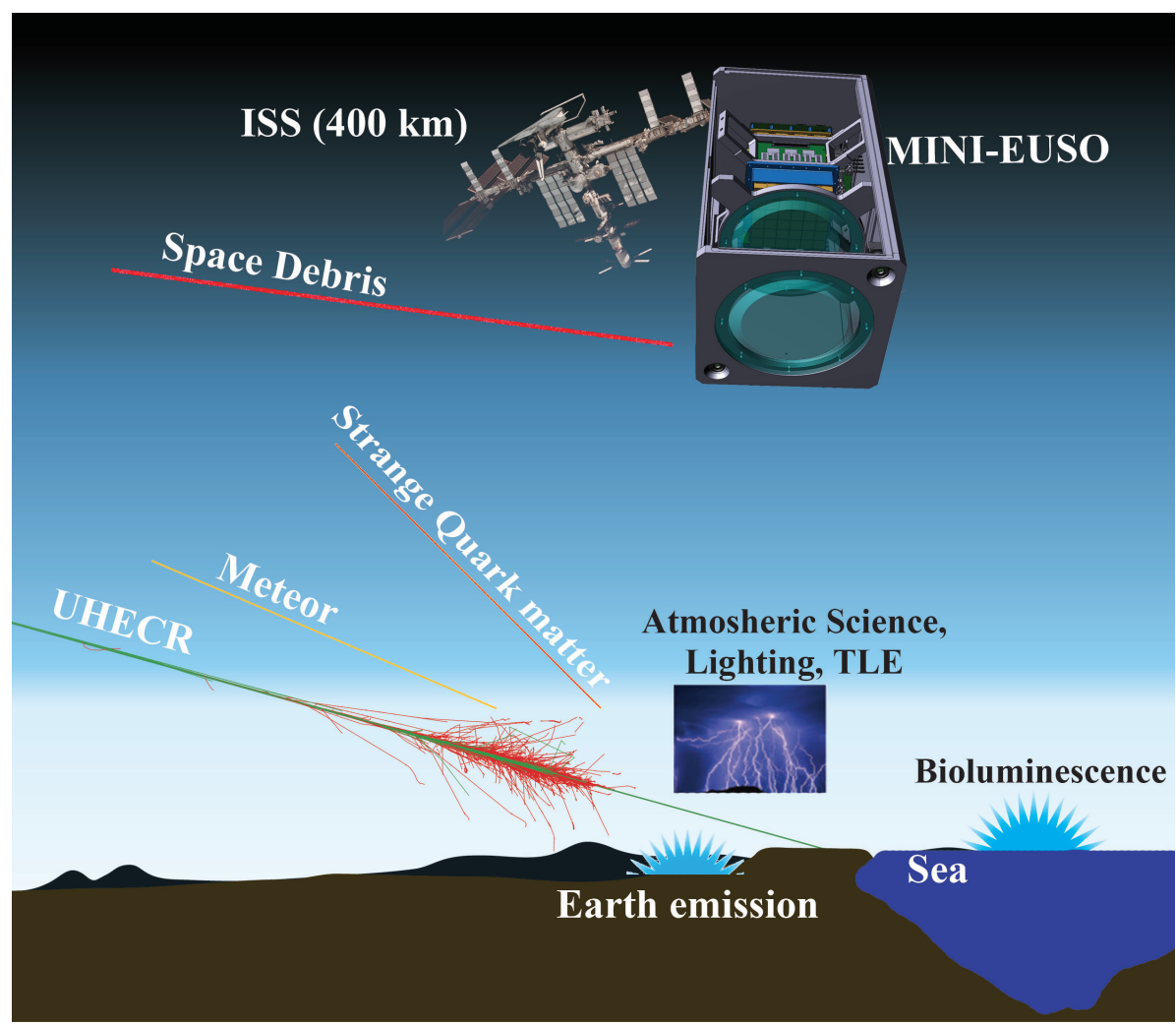

Figure 1: MINI-EUSO science objectives.

The Mini-EUSO space experiment ("UV atmosphere" in Russian Space Program) aims at observing UV light emitting phenomena originating from the Earth (Figure 1). The instrument will be placed in the Russian Zvezda Service Module, looking nadir through the UV transparent window.

The Mini-EUSO instrument[16] comprises a compact telescope with a large field of view $\left(44^{\circ} \times 44^{\circ}\right)$, based on an optical system employing two $25 \mathrm{~cm}$ diameter Fresnel lenses (focal length $30 \mathrm{~cm}$ ) for increased light collection[18]. The UV light is focused onto one Photo Detector Module (PDM) of 2304 pixels $[1,14]$ and stored on on-board disks. Launch is foreseen between Autumn 2017 and beginning of 2018 in the framework of the next manned ASI (Italian Space Agency) flight. Observations are supposed to continue with Russian cosmonauts for several years. The observations will be performed on $\mu$ s to seconds scales both in triggered modes (separately for fast and slow events)[10] and a "continuous mode" consisting of data taken in regular intervals[4]. Through UV Earth observation, Mini-EUSO will allow to study a diverse range of scientific phenomena including atmospheric physics events, strange quark matter, bioluminescence and UHECRs. Furthermore, Mini-EUSO is the first step in a roadmap of potential space debris removal via laser ablation [3]. Data will be saved on data cards to be analysed off-line[9]. In this work we describe the physics objectives of the mission. 


\section{Search for Strange Quark Matter and study of Meteoroids}

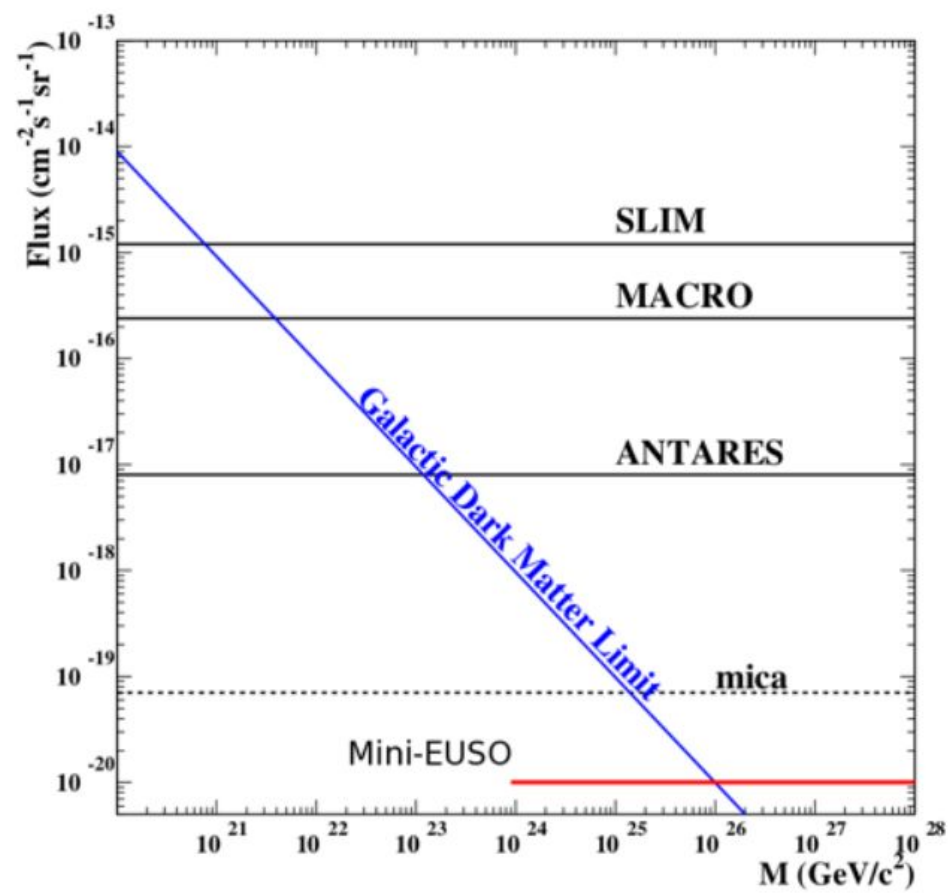

Figure 2: Upper limit to Strange Quark Matter with Mini-EUSO after $24 \mathrm{~h}$ observation as compared to current data.

Strange Quark Matter (SQM), or "Strangelet", is one of the Dark Matter (DM) candidates. SQM, or nuclearities is believed to be a special bound and stable form of quark matter consisting of up, down and strange quarks. The observational strategy developed to detect meteors and meteorites may also be applied to the detection of nuclearites. Since they come from interstellar space, nuclearites have higher velocities than meteors, a wider range of possible trajectories, but move well below the speed of light and trigger as slow events. In just a few hours of observational time Mini-EUSO will be able to set a new lower limit on the detection of Strangelets or provide the first experimental evidence for the existence of Strange Quark Matter (Figure 2).

Meteors, meteorites and fireball observations are key to the derivation of both the inventory and physical characterization of small solar system bodies orbiting in the vicinity of the Earth. For several decades, observation of these phenomena has only been possible through ground-based instruments. Mini-EUSO has the potential to become one of the first operational space-based platforms to share this capability. In comparison to the observation of extremely energetic cosmic ray events, meteoroid phenomena will appear very similar but in contrast are very slow, since their typical speeds are of the order of a few tens of $\mathrm{km} / \mathrm{s}$. By scaling the JEM-EUSO performance in meteoroid detection, we estimate that Mini-EUSO will be sensitive to meteors and meteorites burning with magnitudes $M<+5$ (see Figure 3)[5, 2]. By combining coincidence detections of meteorites with ground based detectors[8], one should be able to significantly increase the accuracy of the predicted meteorite impact point (if present) and facilitate the recovery of fragments. 


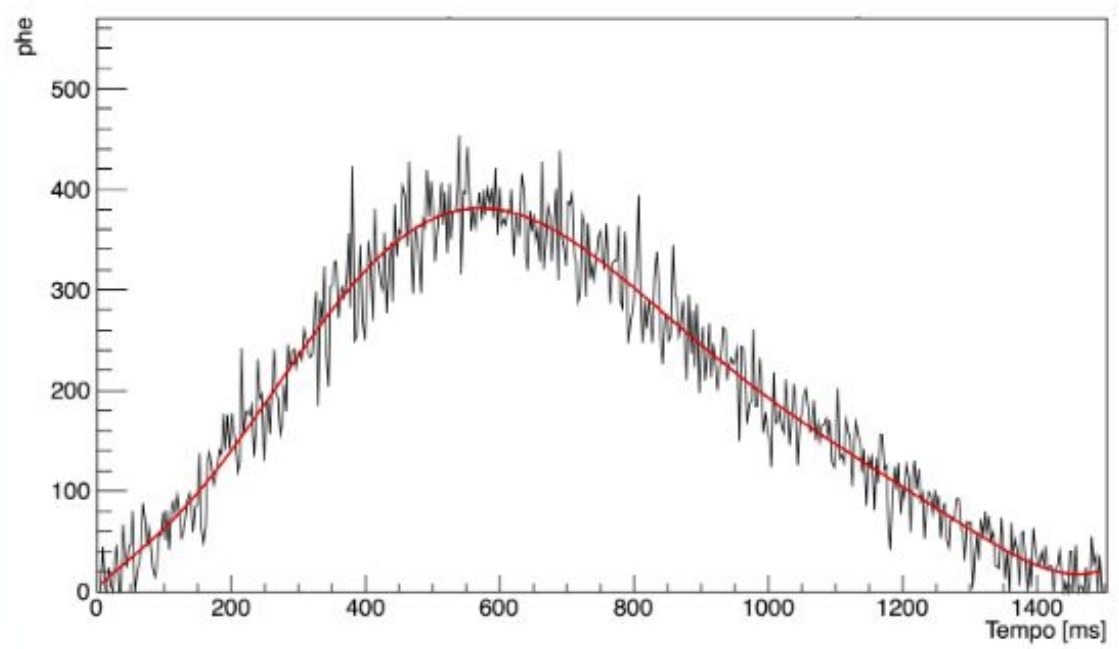

Figure 3: Simulation of a light profile (photoectrons) vs time (ms) of a $\mathrm{M}=+3$ meteor observed by MiniEUSO in the UV. The NIR and visible cameras[17] will also provide information in those spectral ranges.

\subsection{Ultra High Energy Cosmic Rays observation}

Given the size of the lenses it will only be possible to look for $E>10^{21} \mathrm{eV}$ events (see Figure 4), therefore putting only an upper limit on the flux of UHECR. Nonetheless the $\mu$ s triggering capability will be present in Mini-EUSO, allowing to look to this and other classes of unknown phenomena. Furthermore, lasers from the ground will be shot in the field of view of Mini-EUSO when the ISS is passing above. Laser light will simulate the energy and profile of a cosmic ray shower and thus will be used to calibrate the performance of the detector end-to-end. It will also be used to synchronize the on board clock of the system. The uoward-going light generated by the laser will also be used to improve simulations of upward-going neutrino-induced showers.
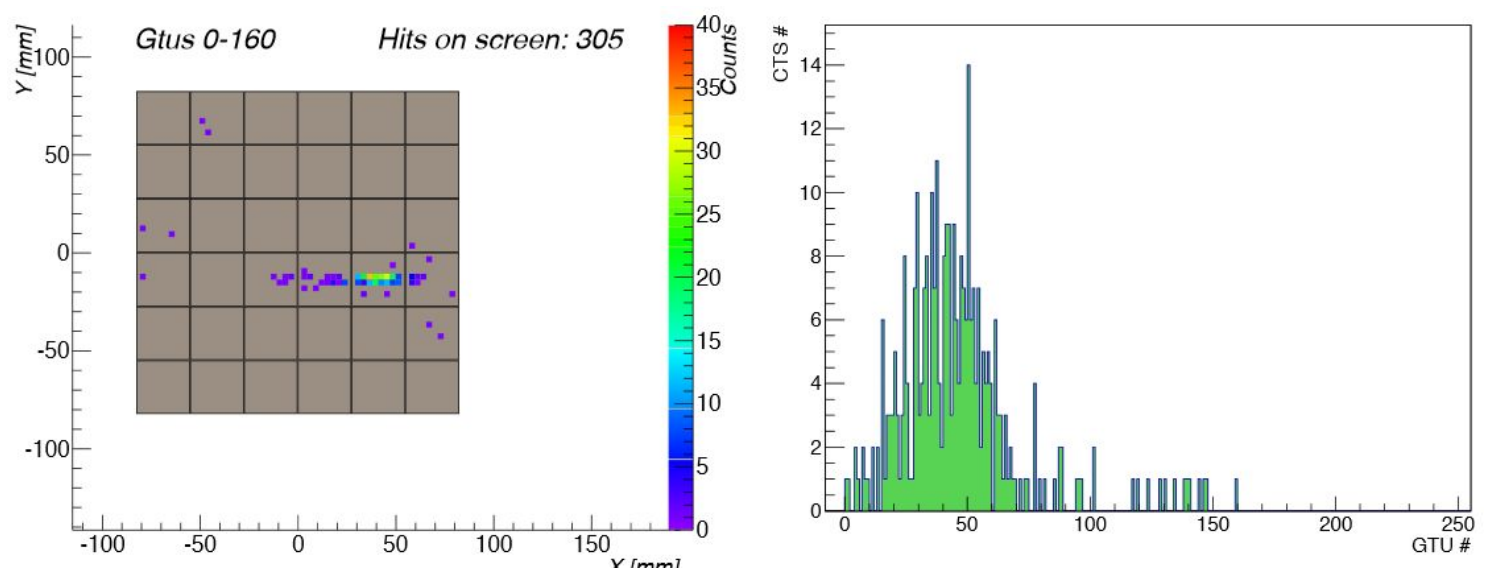

Figure 4: Left panel: Light profile (photoelectrons) observed in the Mini-EUSO focal surface for a $E=10^{21}$ $\mathrm{eV}$ event (simulation). Right panel: number of photoelectrons vs time ( $\mu \mathrm{s})$ for the same event. 


\section{Search and observation of Space Debris}

Another relevant issue would be the observation of space debris. For more than 50 years of space flight, over 30000 tons of satellites and rockets have been launched into space. It is estimated that almost 3000 tons of non-functioning space debris remain in Low Earth Orbit (LEO) in varied forms, ranging from small fragments to large scale structures such as switched off rocket bodies and fully intact multi-ton satellites. Since their orbital velocities are very high, collisions can involve relative impact velocities of the order of $10 \mathrm{~km} / \mathrm{s}$, even the fragments with kinetic energies greater than MJ may cause severe or even catastrophic damage to functioning satellites such as the ISS. Mini-EUSO could be used as a prototype system for tracking such space debris. A wide field-ofview telescope (such as JEM-EUSO) and a novel high efficiency fibre-based laser system (Coherent Amplifying Nework, CAN) could constitute a very useful orbiting debris remediation system.

EUSO detector family and related technology can be used to develop a purely space-based system to solve the growing problem of space debris. Combining a EUSO-class detector with a high-power CAN laser it will be possible to track down and deorbit the most dangerous space debris, around the size of one centimeter, from a distance of up to $100 \mathrm{~km}$. [3] The intense laser beam focused on the debris will produce high-velocity plasma ablation, and the reaction force will reduce the orbital velocity of objects, leading to their reentry into the earth's atmosphere. In the final configuration the system will consist of two major elements:

- The EUSO telescope, which will be used to find debris, was originally planned to detect ultraviolet light emitted from air showers produced by ultra-high energy cosmic rays entering the atmosphere at night. During twilight, thanks to EUSO's wide field of view and powerful optics, it can be used for the detecting high-velocity debris approaching to a space craft.

- The CAN laser, which was originally developed for particle accelerators. It consists of bundles of optical fibers that act in concert to efficiently produce powerful laser pulses. It may achieve both high power of $500 \mathrm{~kW}$ in a 10 seconds burst and a high repetition rate of 105 $\mathrm{Hz}$, which are necessary specifications for a removal operation of a space debris.

Initial proof of concept will employ Mini-EUSO to detect debris from the ISS. We will look for debris tracked by Norad and expected to appear in the field of view of Mini-EUSO and debris not tracked by Norad. Both will be identified by their unique velocity signature (from $\simeq 7$ to 14 $\mathrm{km} / \mathrm{s})$.

\section{Study of UV Emission of Night-Time Earth}

The main scientific objective of the Mini-EUSO will be to map for the first time the Earth's surface at night in the ultraviolet (UV) band (300 - $400 \mathrm{~nm}$ ) with high resolution. The UV band has never previously been studied at night in high resolution due to the difficulties of the measurement requiring a high efficiency of photon detection and a high quality of the optical system, thus not possible with conventional lenses. Data from the Russian Tatiana satellite [6], equipped with a single photomultiplier, but no lenses, resulted in a map with a resolution of the order of $100-200$ $\mathrm{km}$, since this was the field of view of the photomultiplier used. Other measurements by balloonborne detectors have allowed for the study of the night background on smaller spatial scales (tens 
of kilometres), but in very limited geographic areas. Mini-EUSO will provide an accurate map with a spatial resolution of $\simeq 6 \mathrm{~km}$ in timescales of up to $2.5 \mu \mathrm{s}$ and covering all of the planet in the latitudinal range covered by the ISS $\left( \pm 51.6^{\circ}\right)$. Mini-EUSO will also allow for a comparative study between the UV background emitted from airglow[13] and the various surface conditions (mountains, desert, snow, lakes, cities) allowing to quantify, more accurately, the respective the respective anthropic and biologic contributions in the $300-400 \mathrm{~nm}$ frequency band. In the case of the long-term observations ( 3 to 5 years) it will also be possible to probe seasonal variations, related to human activity and/or climate change related to the UV emission. A simulation of the UV background measurements by Mini-EUSO is shown in Figure 5.

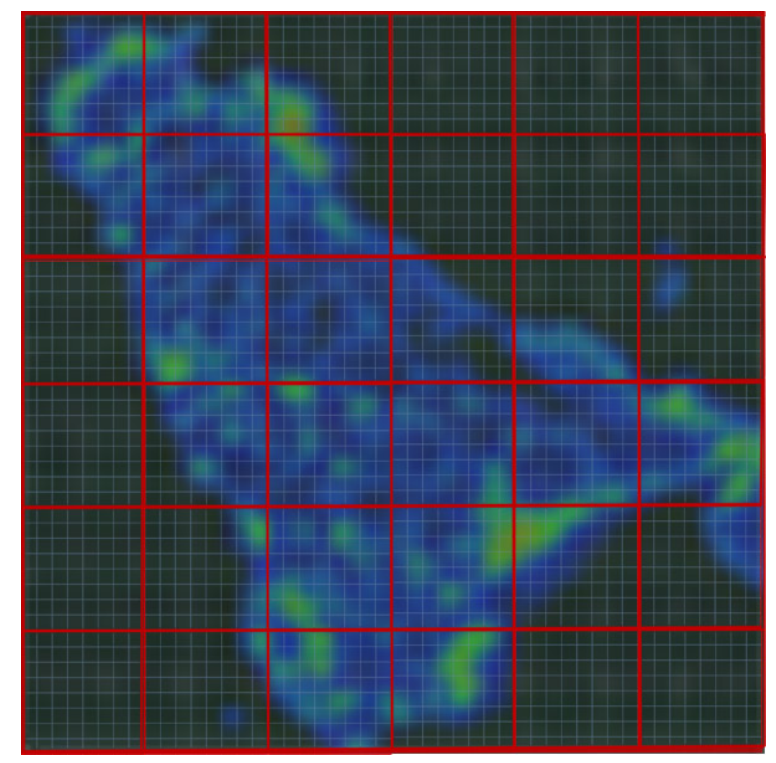

Figure 5: The Italian island of Sicily as it will look from the Mini-EUSO UV measurements (simulated results).

The observation of the atmosphere in the ultraviolet band of the night has never been carried out from space with such a high spatial or temporal resolution. The observations of Mini-EUSO will open several observational windows regarding the presence and evolution of various nocturnal gases such as ozone, water vapour and aerosols.

It will be possible to study the large-scale presence and evolution of the phenomenon of bioluminescence produced by algae, plankton and other such organisms to gain information and knowledge of the state of pollution of the seas. Several effects such as the 'white sea' phenomenon have been observed in the visible range. Similar studies will be carried out by studying land vegetation.

\section{Transient Luminous Events}

Discovered in the late eighties, Transient Luminous Events (TLEs), such as red sprites, elves and blue jets are still poorly known. These phenomena, occurring in the upper atmosphere, have been widely studied in recent years. Previous satellite missions $[6,7,12]$ indicate a high luminosity 
in UV wavelengths and high frequency of TLEs (especially weak ones). Also more recent TLEdevoted instruments such as SMILES (sub millimeter [15]) and ASIM [11] will be complemented by studying TLE events on the micro-second timescale. The spatial resolution of a few km together with the time resolution of microseconds can provide important data for the understanding of the mechanisms of the formation of the filamentary structures of the plasma, especially in the "streamer beard" of the sprite. This should allow for the understanding of the structures of the plasma and the processes that occur in its interior.

\section{Technological Objectives}

Besides the scientific objectives described in the previous section, Mini-EUSO will also address some important issues from the technological point of view:

- First use of wide-area Fresnel lenses in space. Lenses of one square meter have been built by the EUSO collaboration and flown flown as the EUSO-Balloon mission under a stratospheric balloon from Timmins, Canada. Furthermore, a second balloon flight flew with a similar lens system from Wanaka New Zealand for a Ultra-Long duration flight (NASA-SPB) in 2017[19]. Fresnel lenses have been used in different contexts but - to our knowledge - this is the largest Fresnel-optic telescope to be flown in space.

- Optimization and validation of JEM-EUSO observational scheme. As already mentioned, the observation of night time Earth emission under various lighting conditions and moon phases, will greatly improve, through definition of special observing conditions, the performance of larger, JEM-EUSO class telescopes through a more detailed understanding of the background of the Earth.

- Raising of the Technological Readiness Level (TRL) of the JEM-EUSO instrumentation, a typical parameter in the development of devices to be space qualified;

- Test and R\&D of advanced solutions for future space missions, such as studies on the development of SiPM (Silicon Photomultiplier) based photosensors arrays for space applications.

\section{Acknowledgments}

This work was partially supported by Basic Science Interdisciplinary Research Projects of RIKEN and JSPS KAKENHI Grant (22340063, 23340081, and 24244042), by the Italian Ministry of Foreign Affairs and International Cooperation, by the Italian Space Agency through the ASI INFN agreement n. 2017-8-H.0, by NASA award 11-APRA-0058 in the USA, by the Deutsches Zentrum für Luft- und Raumfahrt, by the French space agency CNES, the Helmholtz Alliance for Astroparticle Physics funded by the Initiative and Networking Fund of the Helmholtz Association (Germany), and by Slovak Academy of Sciences MVTS JEM-EUSO as well as VEGA grant agency project 2/0132/17. Russia is supported by the Russian Foundation for Basic Research Grant No 1629-13065-ofi-m, by ROSCOSMOS, by contract 2016-1-U.0, and the Olle Engkvist Byggmästare Foundation.

We dedicate this proceeding to Yoshiya Kawasaki and Jacek Karczmarczyk, who passed away in 2016. 


\section{References}

[1] A.Belov. Mini-EUSO photodetector module data processing system. These proceedings.

[2] Adams, J. H., et al. JEM-EUSO: Meteor and nuclearite observations. Experimental Astronomy, 40:253-279, November 2015. doi:10.1007/s10686-014-9375-4.

[3] Ebisuzaki, T., et al. Demonstration designs for the remediation of space debris from the International Space Station. Acta Astronautica, 112:102-113, 2015.

[4] F.Capel. Mini-EUSO flight software and operations on ISS. These proceedings.

[5] Fenu, F. Simulations of Mini-EUSO observations of UV phenomena in the atmosphere. These proceedings.

[6] Garipov, G. K., et al. UV radiation from the atmosphere: Results of the MSU "Tatiana" satellite measurements. Astroparticle Physics, 24(4):400-408, 2005.

[7] Garipov, G. K., et al. Program of transient UV event research at Tatiana-2 satellite. Journal of Geophysical Research: Space Physics, 115(A5), 2010. ISSN 2156-2202. doi:10.1029/2009JA014765. A00E24.

[8] Kajino, F. Study of Fast Moving Nuclearite and Meteorite using High Sensitivity CMOS Camera with EUSO-TA. These proceedings.

[9] L.Piotrowski. Mini-EUSO data processing and quasi-real time analysis. These proceedings.

[10] M.Bertaina. The Mini-EUSO multilevel trigger algorithm and its performance. These proceedings.

[11] Neubert, T. et al. ASIM - an Instrument Suite for the International Space Station. In Coupling of Thunderstorms and Lightning Discharge to Near-Earth Space: Proceedings of the Workshop, volume 1118, pages 8-12. AIP Publishing, 2009.

[12] Panasyuk, M. I., et al. Transient luminous event phenomena and energetic particles impacting the upper atmosphere: Russian space experiment programs. Journal of Geophysical Research: Space Physics (1978-2012), 115(A6), 2010.

[13] P.Bobik. Airglow dynamics observations by MINI-EUSO. These proceedings.

[14] Plebaniak, Z. Mini-EUSO / EUSO-SPB HVPS system for *-EUSO detectors. These proceedings.

[15] Randel, W. J. et al. Isolation of the ozone QBO in SAGE II data by singular-value decomposition. Journal of the atmospheric sciences, 53(17):2546-2559, 1996.

[16] Ricci, M. MINI-EUSO, a precursor mission to observe and study Atmosphere and Earth UV emission from the International Space Station. These proceedings.

[17] S.Turriziani. Ancillary detectors for the MINIEUSO telescope: control software development and expected science. These proceedings.

[18] Takizawa, Y. MINI-EUSO optics design and test. These proceedings.

[19] Wiencke, L. EUSO-SPB Mission and Science. These proceedings. 